\title{
Laser-plasma electron acceleration in dielectric capillary tubes
}

\author{
G. Genoud • K. Cassou • F. Wojda • H.E. Ferrari • C. Kamperidis • M. Burza • \\ A. Persson • J. Uhlig • S. Kneip • S.P.D. Mangles • A. Lifschitz • B. Cros • \\ C.-G. Wahlström
}

Received: 25 January 2011 / Revised version: 19 April 2011 / Published online: 29 July 2011

(C) The Author(s) 2011. This article is published with open access at Springerlink.com

\begin{abstract}
Electron beams and betatron X-ray radiation generated by laser wakefield acceleration in long plasma targets are studied. The targets consist of hydrogen filled dielectric capillary tubes of diameter 150 to 200 microns and length 6 to $20 \mathrm{~mm}$. Electron beams are observed for peak laser intensities as low as $5 \times 10^{17} \mathrm{~W} / \mathrm{cm}^{2}$. It is found that the capillary collects energy outside the main peak of the focal spot and contributes to keep the beam self-focused over a distance longer than in a gas jet of similar density. This enables the pulse to evolve enough to reach the threshold for wavebreaking, and thus trap and accelerate electrons. No electrons were observed for capillaries of large diameter $(250 \mu \mathrm{m})$, confirming that the capillary influences the interaction and does not have the same behaviour as a gas cell. Finally, X-rays are used as a diagnostic of the interaction
\end{abstract}

G. Genoud · C. Kamperidis · M. Burza · A. Persson .

C.-G. Wahlström $(\bowtie)$

Department of Physics, Lund University, P.O. Box 118, 22100

Lund, Sweden

e-mail: claes-goran.wahlstrom@fysik.lth.se

Fax: +46-46-2224250

K. Cassou · F. Wojda · H.E. Ferrari · A. Lifschitz · B. Cros Laboratoire Physique Gaz et Plasmas (UMR 8578), Université

Paris-Sud 11, 91405 Orsay Cedex, France

H.E. Ferrari

CONICET, Buenos Aires, Argentina

J. Uhlig

Department of Chemical Physics, Lund University, P.O. Box 124,

22100 Lund, Sweden

S. Kneip · S.P.D. Mangles

Blackett Laboratory, Imperial College London, London,

SW7 2BZ, UK and, in particular, to estimate the position of the electrons trapping point inside the capillary.

\section{Introduction}

When an intense femtosecond laser pulse interacts with gas, a plasma is formed and the ponderomotive force of the pulse generates a large amplitude plasma wave. This wave can break, trap, and accelerate electrons. The acceleration field can be more than a thousand times higher than those achieved in accelerators based on conventional technology. Laser plasma wakefield accelerators are thus a promising alternative to conventional accelerators, especially because of their compactness and short pulse duration. Quasi-monoenergetic beams of electrons, of hundreds of $\mathrm{MeV}$ kinetic energy, have been produced in several experiments [1-5]. The laser pulse can also be externally guided, for example by using either a plasma channel created by a discharge inside a capillary [6] or a dielectric capillary tube. In this article, we discuss the use of gas-filled dielectric capillary tubes to assist guiding and thereby enable wave breaking and self-trapping of electrons at lower laser intensities than required with gas jets, which also have a homogeneous transverse gas profile.

Even when the intensity at the entrance of the plasma is below the threshold for wave-braking and electron trapping, it can be reached through the non-linear evolution of the pulse, undergoing self-focusing and pulse compression. In the experiment reported here, electron beams were observed with peak laser intensities as low as $I=(5 \pm 2) \times$ $10^{17} \mathrm{~W} / \mathrm{cm}^{2}$ (corresponding to a normalized pulse amplitude $a_{0}=0.5$ ) for a plasma electron density of the order of $10^{18} \mathrm{~cm}^{-3}$. With such a low intensity, the evolution of 
the laser pulse would not be sufficient over the length typical for a gas jet experiment (a few $\mathrm{mm}$ ). However, capillary tubes longer than $6 \mathrm{~mm}$ provide a distance of interaction long enough to achieve electron trapping and acceleration.

The inside walls of the capillary tubes are smooth at the laser wavelength and the laser pulse is guided by Fresnel reflection on the walls. Ultrashort intense laser pulses propagate using eigenmodes of the capillary tubes [7]. For a Gaussian distribution of energy at the entrance of the capillary, with a waist matched to the capillary radius, more than $98 \%$ of the incident energy can be transferred into the fundamental capillary mode, inducing quasi-perfect monomode guiding [8]. This can produce linear plasma waves over several centimetres [9] which could be used as an accelerating structure for injected electrons. The electron density in this type of capillary can be arbitrarily low which is an advantage, as the dephasing length can then be made equal or longer than the capillary tube. Dephasing occurs at the point where the electrons outrun the plasma wave and start decelerating. The dephasing length is given by $L_{d}=\left(\omega_{0} / \omega_{p}\right)^{2} \lambda_{p}$, where $\omega_{0}$ is the laser frequency, $\omega_{p}=\left(n_{e} e^{2} / \epsilon_{0} m_{e}\right)^{1 / 2}$ the plasma frequency of a plasma of electron density $n_{e}$ and $\lambda_{p}$ the corresponding plasma wavelength. $e$ and $m_{e}$ are the charge and mass of an electron, respectively. However, when the capillary diameter is too large to match the focal spot, the propagation is not monomode, as higher order modes are then also excited. In the experiment reported here, the focal spot size was deliberately chosen significantly smaller than the tube diameter to allow higher order modes to be excited. This resulted in a regime where external guiding, non-linear pulse evolution and self trapping take place inside the tube.

$\mathrm{X}$-rays can be produced within a wakefield accelerator through a process called betatron oscillations [10]. Transverse fields can cause electrons within the wakefield to oscillate transversely to their acceleration direction and produce X-rays, which have great potential as novel ultra-short radiation sources. Moreover, their observation provides insight into the laser-plasma interaction itself, because their production is directly linked to the trapping and acceleration of electrons. For large amplitude oscillations of electrons, the radiation emitted close to the axis can be shown [11] to have a synchrotron-like spectrum, which is a broadband spectral distribution peaked close to the critical energy $E_{c}=3 / 2 \hbar K \gamma^{2} \omega_{\beta}$, with $\gamma m_{e} c^{2}$ the electron energy [12]. The betatron frequency $\omega_{\beta}=\omega_{p} / \sqrt{2 \gamma}$ and the betatron strength parameter $K=\gamma r_{\beta} \omega_{\beta} / c$ describe the trajectory of an electron oscillating with an amplitude $r_{\beta}$.

\section{Experiment}

\subsection{Experimental arrangement}

The experiment was performed using the $10 \mathrm{~Hz}$ multiterawatt Ti:sapphire laser system at the Lund Laser Centre,

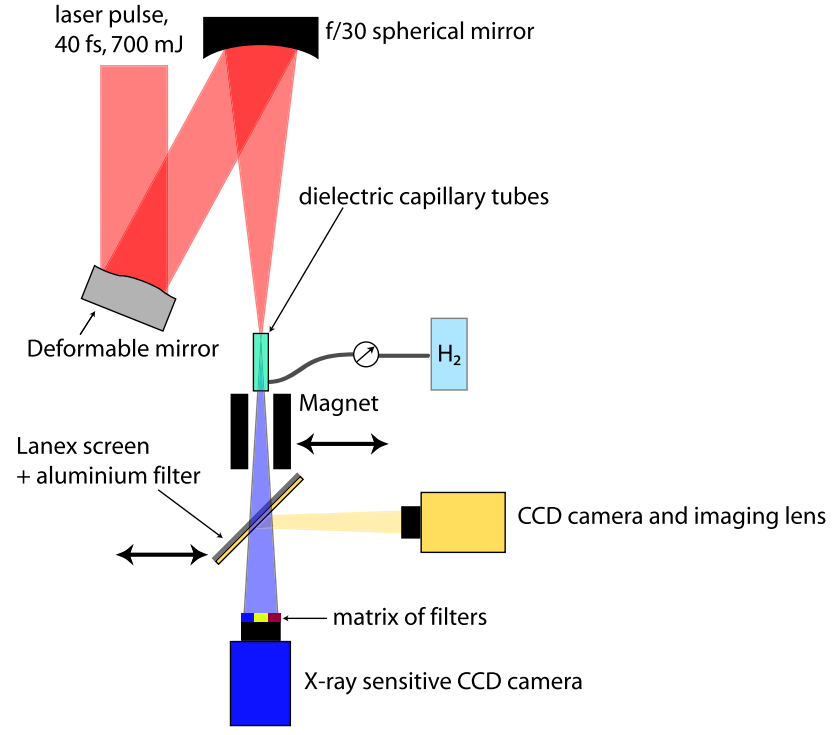

Fig. 1 Schematic view of the experimental setup

which delivered $0.7 \mathrm{~J}$ within a focal spot of $50 \mu \mathrm{m}$ size (radius of the first minimum of an Airy-like pattern) in $40 \mathrm{fs}$ (FWHM) pulse duration at $\lambda_{0}=795 \mathrm{~nm}$ centre wavelength. A schematic view of the experimental arrangement is presented in Fig. 1. The laser pulses were loosely focused at the entrance of dielectric capillary tubes, using a spherical mirror in combination with a deformable mirror, to correct aberrations of the phase front in the focal plane. Glass capillary tubes with inner diameter ranging from $d=150 \mu \mathrm{m}$ to $250 \mu \mathrm{m}$ and length varying between $6 \mathrm{~mm}$ and $20 \mathrm{~mm}$ were aligned along the optical axis. Hydrogen gas was filled into the tubes through two thin $(\sim 300 \mu \mathrm{m})$ slits located $2.5 \mathrm{~mm}$ from each end of the tubes. An electro-magnetic valve controlled the pressure in a reservoir which in turn controlled the flow of gas into the capillary tube. Gas densities inside the capillary corresponding to electron densities in the range $5 \times 10^{17}-1.5 \times 10^{19} \mathrm{~cm}^{-3}$, were achieved and characterized by off-line interferometric measurement. Each capillary tube could be used for a large number of laser shots ( $\sim 100$ shots in the same capillary), as long as the focused laser beam remained well centered at the capillary entrance. Pointing variations due to thermal drifts and mechanical vibrations were therefore minimized or actively compensated for [13]. The laser pulse was guided in the capillary in the multi-mode regime [7], ionizing the gas and producing a wake. Figure 2 illustrates how the capillary guides the laser beam in vacuum. In (a), the focal spot at the entrance of the capillary tube is shown. In (b), the mode pattern at the exit of the capillary tube shows that the laser pulse was guided in the multi-mode regime. The transmission within the capillary diameter is $70 \%$ (capillary of $20 \mathrm{~mm}$ long and $200 \mu \mathrm{m}$ diameter). The symmetry of this multi-mode structure at the exit of the capillary tube is the criterion used for the alignment of the tube on the laser axis. 
Electrons from the plasma were trapped, accelerated and then detected on a phosphor screen (Kodak Lanex), placed $59 \mathrm{~cm}$ after the laser focus, imaged by a CCD camera. A permanent magnet $(B=0.7 \mathrm{~T}$, length $100 \mathrm{~mm}$ ) could be moved into position between the capillary exit and the phosphor screen to deflect the electrons and thereby allow their energy distribution to be measured. Electrons with energy below $40 \mathrm{MeV}$ did not reach the phosphor screen and were thus not detected. Far-field profiles of the X-rays, generated due to betatron oscillations inside the capillary, were recorded by a 16 bit CCD X-ray camera placed on the laser axis. X-rays and electron spectra were recorded simultaneously when the electron beam was deflected from the axis by the magnet. A matrix of metallic filters was placed over the chip of the camera in order to block laser and visible light and to provide a low resolution measurement of the X-ray spectrum. The camera, an Andor 434-BN CCD chip with $1024 \times 1024$ pixels of $13 \mu \mathrm{m}$ size, was placed $89 \mathrm{~cm}$ away from the source, corresponding to a chip collection angle of $15 \times 15 \mathrm{mrad}^{2}$.

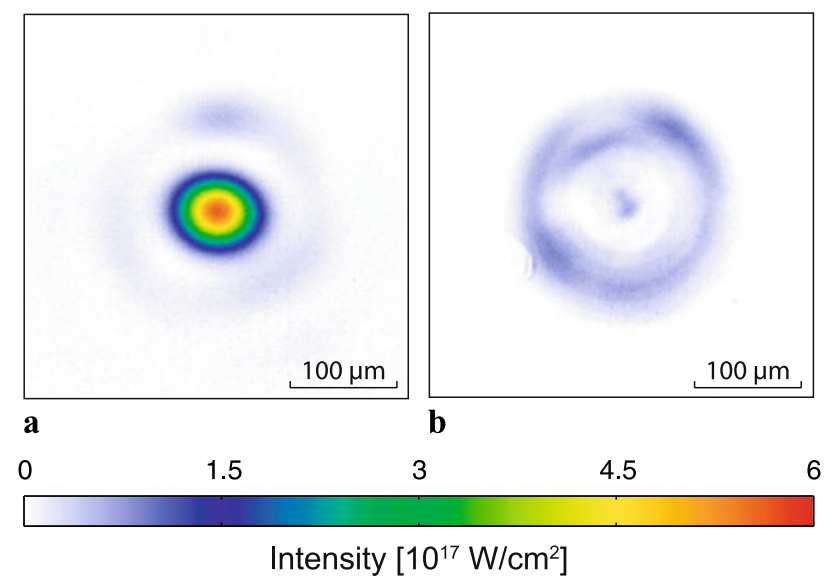

Fig. 2 Transmission trough a capillary tube of $20 \mathrm{~mm}$ long and $200 \mu \mathrm{m}$ diameter in vacuum. In (a) the focal spot at the entrance of the capillary tube and (b) the multi-mode pattern at the exit of the capillary tube. The transmission within the capillary diameter is $70 \%$

\subsection{Electrons}

Electron beams, with charge varying in the range 1-100 pC, were observed. The quality of the beam was found to depend very sensitively on the plasma electron density. Individual shots showed peaks with energies up to $170 \mathrm{MeV}$, with energy spreads as low as $7 \%$ and divergence as low as 4 mrad FWHM. At optimum plasma density, monoenergetic features were observed, as illustrated in Fig. 3(a) for $n_{e}=3 \times 10^{18} \mathrm{~cm}^{-3}$, a capillary length of $20.5 \mathrm{~mm}$ and a diameter of $200 \mu \mathrm{m}$. At higher densities, trapping occurs at more than one point leading to broad electron spectra, occasionally with multiple peaks as shown in Fig. 3(b) for $n_{e}=8 \times 10^{18} \mathrm{~cm}^{-3}$ in a capillary $11 \mathrm{~mm}$ long and of $200 \mu \mathrm{m}$ diameter. Both spectra were recorded at a peak laser intensity of $6 \times 10^{17} \mathrm{~W} / \mathrm{cm}^{2}$.

The influence of the plasma electron density, laser intensity and capillary parameters, such as diameter and length, are presented in Fig. 4. In (a), the charge above $40 \mathrm{MeV}$ is plotted as a function of the plasma electron density for different capillary diameters, and laser intensities. The capillary length was kept fixed $(20.5 \mathrm{~mm})$ and the diameter varied: $180 \mu \mathrm{m}$ (circles), $200 \mu \mathrm{m}$ (squares, diamonds) and $250 \mu \mathrm{m}$ (triangles). The laser intensity was $6 \times 10^{17} \mathrm{~W} / \mathrm{cm}^{2}$ except for the data represented by squares, where the laser intensity was $5 \times 10^{17} \mathrm{~W} / \mathrm{cm}^{2}$. Figure 4(a) shows that for each capillary diameter and laser intensity, there is a narrow range of electron density for which accelerated electrons are produced. In this regime of acceleration, the plasma electron density is a crucial parameter for the trapping and acceleration process. This is also observed in simulations where changes in diameter and intensity significantly change the pulse evolution, as discussed by Ferrari et al. in [14]. In order to fully understand this very complex interaction and, in particular, how the different parameters influence the value of the optimum density, further modelling and systematic studies are required. Here, we merely demonstrate this sensitivity. Finally, for capillaries of $250 \mu \mathrm{m}$, diameter no electrons were observed over the entire range of electron densities explored. This suggests that for such large diameter
Fig. 3 Experimental electron spectra. In (a) a peaked spectrum and in (b) a broad spectrum with multiple peaks, with a total charge above $40 \mathrm{MeV}$ of $2.3 \mathrm{pC}$ and $7.7 \mathrm{pC}$, respectively
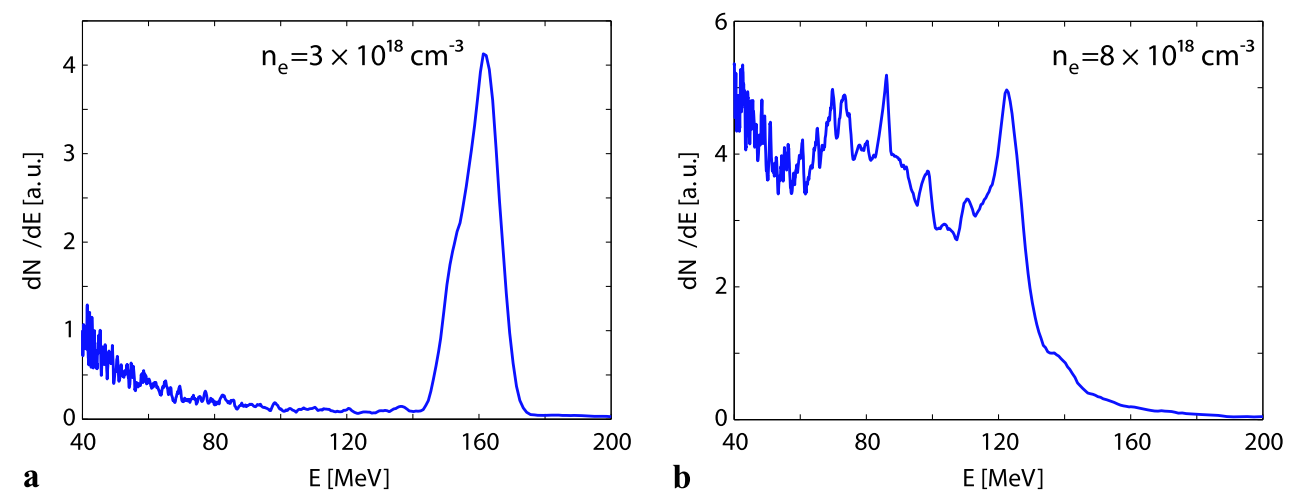

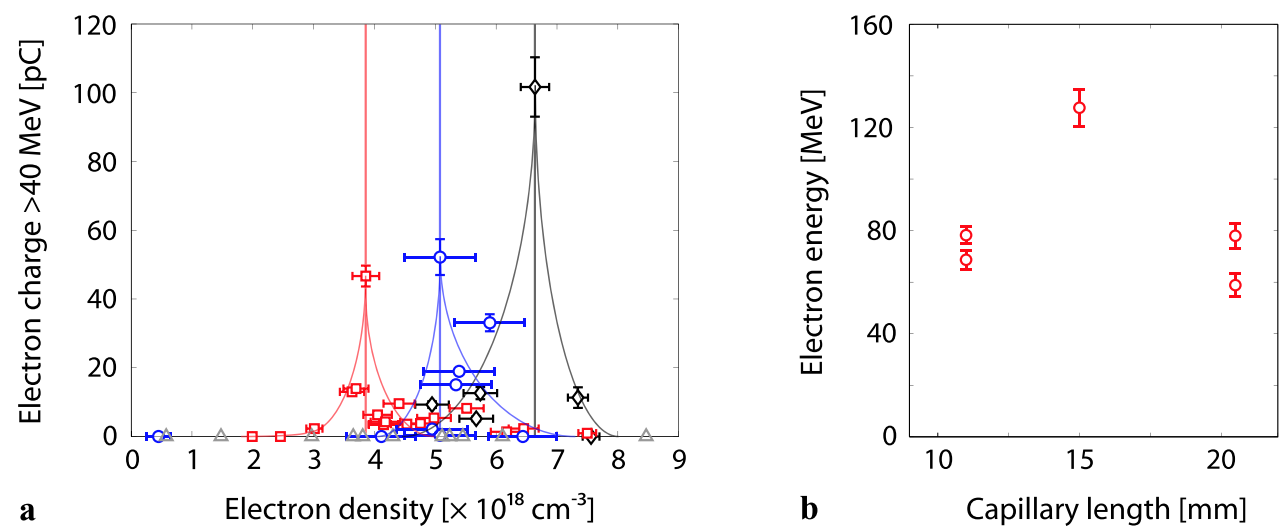

Fig. 4 Influence of the electron density, the laser intensity and the capillary parameters (diameter and length) on the electron beams. In (a) the charge above $40 \mathrm{MeV}$ as function of electron density is shown for different capillary diameters: $180 \mu \mathrm{m}$ (blue circles), $200 \mu \mathrm{m}$ (red squares, black diamonds) and $250 \mu \mathrm{m}$ (grey triangles) for a laser intensity of $6 \times 10^{17} \mathrm{~W} / \mathrm{cm}^{2}$ except for the points represented by the squares

the capillary acted mainly as a windowless gas cell: the reflection from the wall are not sufficient to contribute significantly to the pulse evolution. In Fig. 3(b), the peak energy as a function of the capillary length is shown at a fixed electron density of $5 \times 10^{18} \mathrm{~cm}^{-3}$ for a capillary diameter of $180 \mu \mathrm{m}$ and laser intensity of $6 \times 10^{17} \mathrm{~W} / \mathrm{cm}^{2}$. The onset of dephasing is observed as the peak energy decreases for the longest capillaries. At this density the theoretical dephasing length is $L_{d}=5 \mathrm{~mm}$. As it is seen in the simulations (see, for example Fig. 8), for the values of densities and power considered, self-focusing is a slow process: it takes $6.4 \mathrm{~mm}$ in this example for $a_{0}$ to reach an adequate value for electron injection and trapping. Adding the evolution distance to the dephasing length gives a value of the order of what we observe in the experiment. Thus, the maximum electron energy can be reached inside the capillary. Alternatively, pump depletion can also contribute to limit the maximum energy. From simulations (Fig. 6 of [14]), it is estimated that $40 \%$ of the laser energy is depleted after $11 \mathrm{~mm}$, where self-focusing stops.

\subsection{X-rays}

The production of betatron X-rays is directly linked to the trapping and acceleration of electrons. The far-field distribution of X-ray beams were recorded and gave additional insight into the interaction itself. The number of X-ray photons obtained strongly depends on the charge of the electron beam, and thus also on the plasma electron density. We obtained up to $\sim 5 \times 10^{4}$ photons $/ \mathrm{mrad}^{2}$ over the range 1$10 \mathrm{keV}$, which is comparable to the results in [10].

Figure 5(a) and (b) show far-field distributions of the $\mathrm{X}$-ray beam for two slightly different electron densities, where the laser intensity was $5 \times 10^{17} \mathrm{~W} / \mathrm{cm}^{2}$. Each point corresponds to one laser shot. The graph shows complete data sets and therefore also illustrates the shot to shot fluctuations. The lines are drawn to guide the eye. The peak electron energy is plotted as function of the capillary length in (b) for $n_{e}=5 \times 10^{18} \mathrm{~cm}^{-3}$. The effect of dephasing is clearly visible $n_{e}=5.3 \times 10^{18} \mathrm{~cm}^{-3}$ and $n_{e}=5.9 \times 10^{18} \mathrm{~cm}^{-3}$, respectively. Both were produced in a capillary $20 \mathrm{~mm}$ long and of $180 \mu \mathrm{m}$ diameter and recorded using a uniform filter $(6 \mu \mathrm{m}$ mylar and $3 \mu \mathrm{m} \mathrm{Al}$ ) in front of the CCD chip. Figure 5(c) shows a radial profile, averaged over angles (red curve) of the image of Fig. 5(a), as well as line-outs in two transverse directions (black and grey curves) of a simulated $\mathrm{X}$ ray beam, from simulations discussed in the next section, with $n_{e}=5 \times 10^{18} \mathrm{~cm}^{-3}$, a capillary diameter of $150 \mu \mathrm{m}$ and a length of $20 \mathrm{~mm}$ for an X-ray photon energy of $1 \mathrm{keV}$. Figure 5(d) shows the X-ray beam distribution produced in a capillary tube of length $20 \mathrm{~mm}$ and diameter $200 \mu \mathrm{m}$, $n_{e}=7 \times 10^{18} \mathrm{~cm}^{-3}$, and a different set of filters (a base of $6 \mu \mathrm{m}$ mylar and $3 \mu \mathrm{m} \mathrm{Al}$, with a $3 \mu \mathrm{m}$ thick vertical stripe of $\mathrm{Al}$ and a $3 \mu \mathrm{m}$ thick horizontal stripe of $\mathrm{Sn}$ ). Assuming a synchrotron-like spectrum, using the tabulated transmission data of the filters and known CCD sensitivity, an estimate of the critical energy $E_{c}=1.3 \mathrm{keV}$ was deduced using a leastsquares method [15].

The shape of the edge of the beam on the images of Fig. 5 shows that the wall of the capillary in the output plane casts a shadow, indicating that the source of the X-rays is inside the tube. The shadow of the wall is observed also on the simulation results and its size is similar to the experimental data. From the different images in Fig. 5, it is clearly observed that the divergence of the X-ray beam varies, which can be related to a different geometry (different capillary tubes) or a different position of the X-ray source along the capillary axis, due for example to a different electron density.

Sources situated at different points along the axis of the capillary produce circular beams of different radii on the detector, as illustrated in Fig. 6. The points closest to the entrance of the tube, situated on the left in Fig. 6, will therefore produce the smallest beam and the point closest to the exit 
Fig. 5 Far-field distribution of the X-ray beam for two different densities in (a) and (b). In (d) the absorption in additional stripes of metallic foils placed in front of the detector are visible and are use to make a rough estimate of the X-ray spectrum. In (c), radial average profile of (a) (red curve), horizontal (black curve) and vertical (grey curve) line-outs of a simulated $\mathrm{X}$-ray beam profile are shown

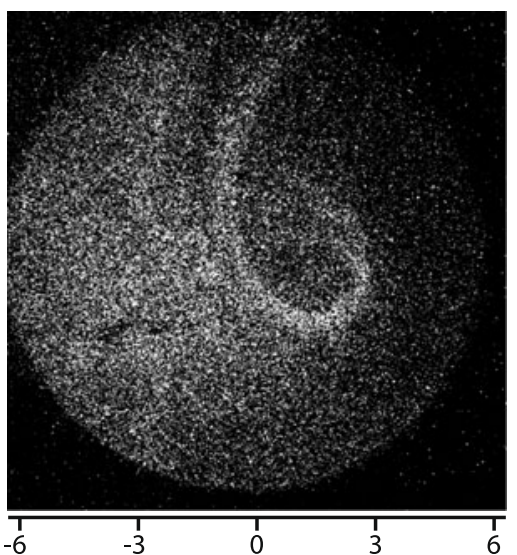

Divergence $x[\mathrm{mrad}]$

a

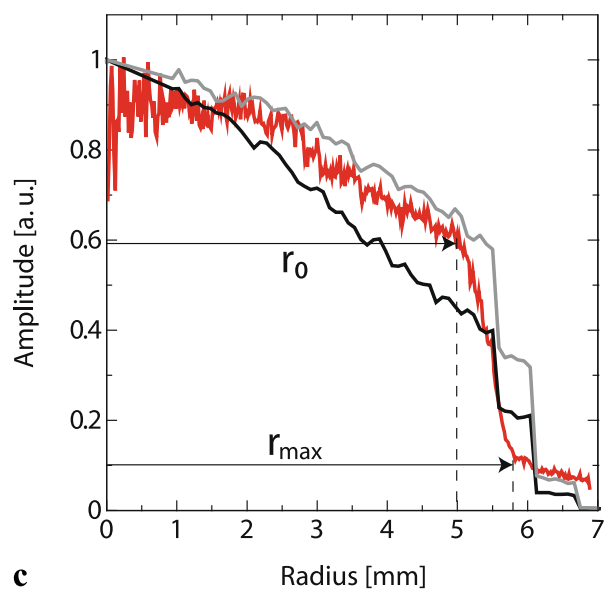

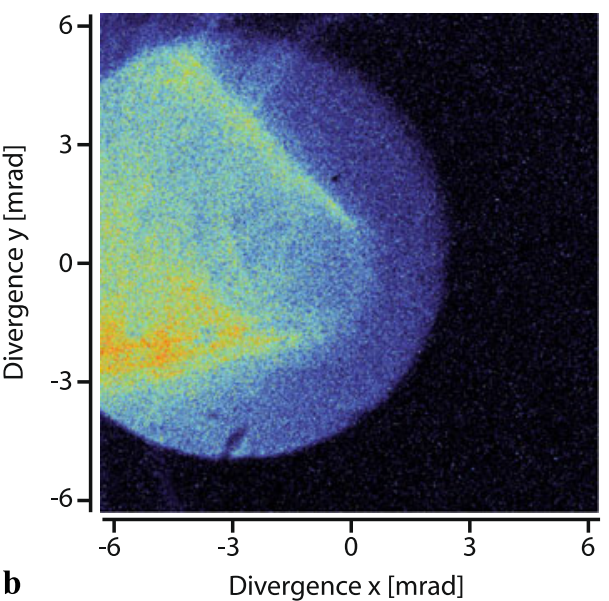

b

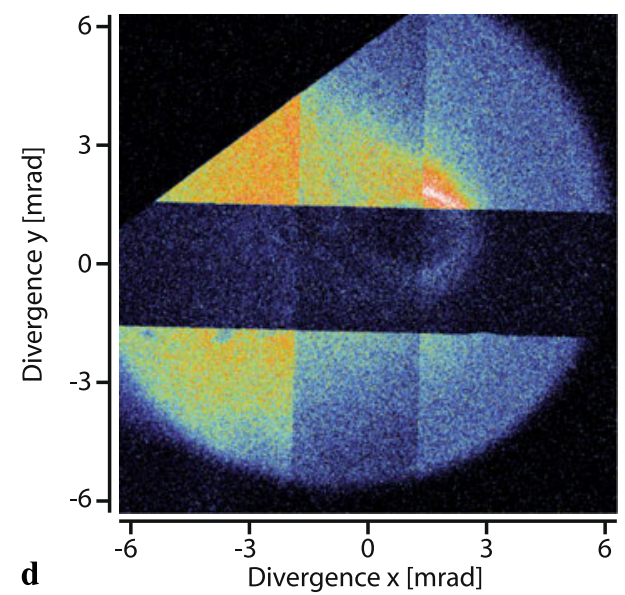

of the tube produces the largest one. This means that the radius, $r_{0}$, where the signal starts to decrease corresponds to emission from the point producing the smallest beam, i.e. the point closest to the entrance of the capillary. As shown in Fig. 5(c), $r_{0}$ can be obtained from the X-ray image and then using simple geometry, the position of the start of the $\mathrm{X}$-ray emission can be estimated. Assuming that X-rays are not reflected by the capillary walls, the distance on axis between the start of the X-ray source and the exit of the capillary, $L_{X}$, can be written as $L_{X}=L /\left[\left(r_{0} / r_{\text {cap }}\right)-1\right]$ where $L$ is the distance between the capillary output and the CCD camera, and $r_{\text {cap }}$ the capillary tube radius. For example in the case of Fig. 5(a), $r_{0}=5 \mathrm{~mm}$, and the distance from the capillary entrance $L_{\text {cap }}-L_{X}$ to the beginning of the X-ray emission is then estimated as $4.6 \mathrm{~mm}$.

The shape of the edge of the beam is also an indicator of the extend of the X-ray source, or of the existence of multiple sources. The radius $r_{\max }$ corresponds to emission from the point producing the largest beam, i.e. the point closest to the exit of the capillary. Using the same calculation as previously, it is possible to estimate where the $\mathrm{X}$-ray emission stops. For the case in Fig. 5(c), we find $r_{\max }=5.8 \mathrm{~mm}$ and the position of the end of the source is then estimated to be
$6.8 \mathrm{~mm}$ from the capillary entrance. The source therefore extends from 4.6 to $6.8 \mathrm{~mm}$ after the entrance of the capillary. However, this is only a lower limit for the extend of the source as the detector is not perfect and the signal drops below the noise level. According to [16-19], the transverse source size is expected to be of the order of a few microns which would result in a radius difference of about $\sim 0.2 \mathrm{~mm}$ on the detector. This has been neglected as the radius difference due to the longitudinal source size is much larger.

As the electrons start emitting X-rays as soon as they are trapped, the position of the X-ray source can be used to determine the trapping position. However, due to the filters in front of the CCD camera, only X-rays above $500 \mathrm{eV}$ were detected and only the point where the electrons have been sufficiently accelerated to produce X-rays above $500 \mathrm{eV}$ can be determined. Figure 7 shows the dependence of the position of the onset of the first X-ray emission, determined from the X-ray beam diameter, on the electron density for different capillaries and laser intensities. We observe that for most of the capillaries the X-ray emission starts at a shorter distance when the density is increased. The capillary diameter, as well as the laser intensity, also influences the result, confirming that the whole acceleration process is very sensitive 


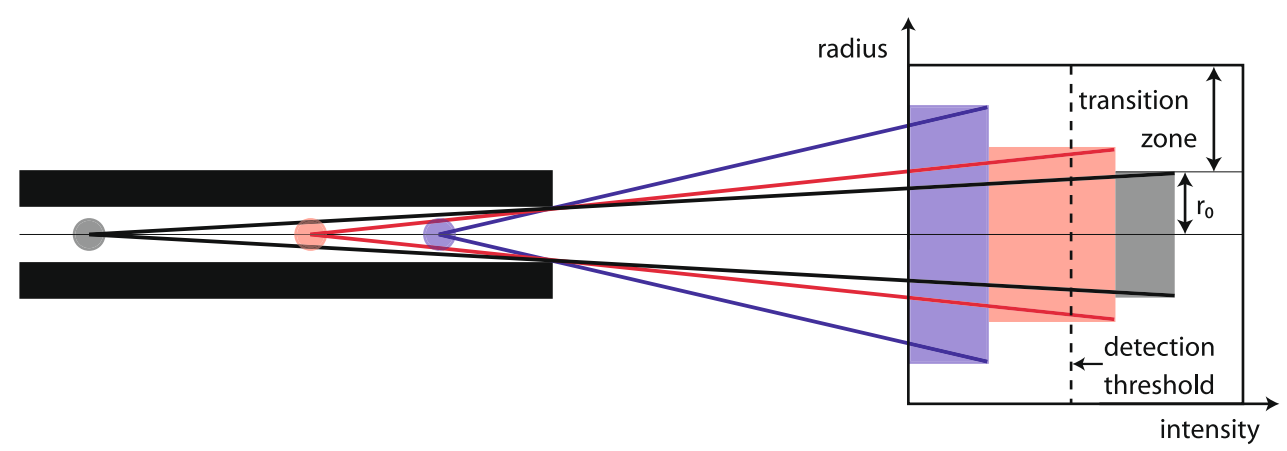

Fig. 6 Schematic explanation of the interpretation of the far-field intensity profiles images of the X-ray beams. The longitudinal spread of the X-ray emission inside the capillary tube is represented as different point sources. Depending on the position of the source, the beams outside of the capillary obtain different divergence. The points closest

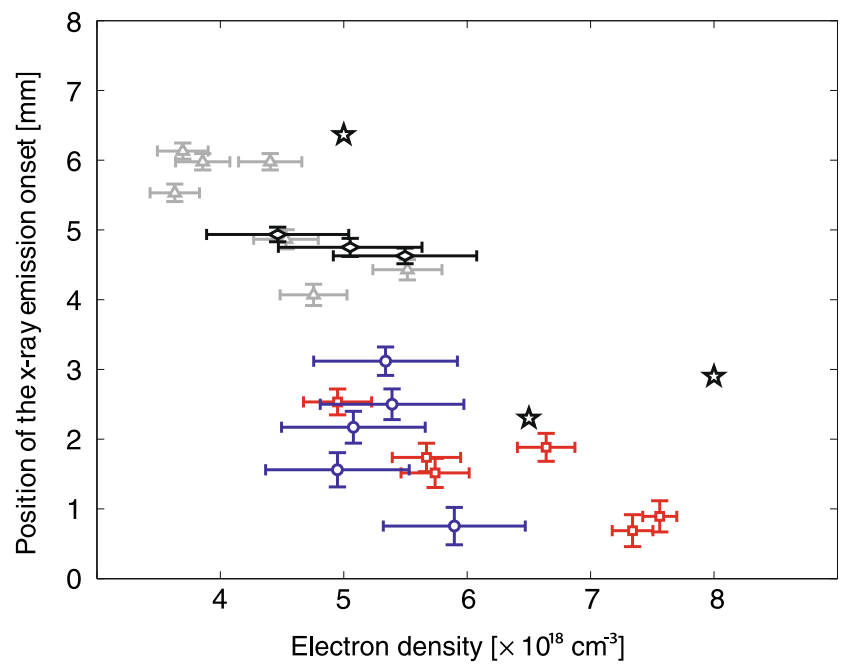

Fig. 7 The diameter of the X-ray image can be used to determine the position of the onset of the first X-ray emission relative to the laser focus inside the capillary. This is done for different capillaries as a function of electron density: a capillary of $20 \mathrm{~mm}$ length and $200 \mu \mathrm{m}$ diameter (grey triangles, red squares), a capillary of $20 \mathrm{~mm}$ length and $180 \mu \mathrm{m}$ diameter (blue circles), a capillary of $15 \mathrm{~mm}$ length and $180 \mu \mathrm{m}$ diameter (black diamonds) for a laser intensity of $6 \times 10^{17} \mathrm{~W} / \mathrm{cm}^{2}$ except for the points represented by the triangles and the diamonds where the laser intensity was $5 \times 10^{17} \mathrm{~W} / \mathrm{cm}^{2}$. Each point corresponds to one laser shot. The stars correspond to the trapping points obtained from the simulations described in the next section

to these parameters. This is consistent with the observations of the electron beam, as shown in Fig. 4(a). The points represented by stars in Fig. 7 correspond to the trapping position obtained from the simulations described in the next section. A trend similar to the experimental one is observed.

Superimposed over a more-or-less uniform background, the far-field beam profiles in Fig. 5(a) and (b) show spatial features, which suggest particular trajectories of the electrons [18]. A "spiral-like" feature is observed in the images for many different shots and for different capillaries. As- to the entrance in the tube, situated on the left of the figure, produce the smallest radius on the detector. By measuring $r_{0}$, it is possible to estimate the start of the X-ray emission and therefore the trapping point of the electrons

suming that specular reflections of X-rays from the inside walls are negligible, the spiral shapes might be understood as the X-ray emission characteristics of electrons along a spiral-like trajectory [20]. Features similar to the observed ones correspond to an oscillation amplitude of the order of $\sim 0.1 \mu \mathrm{m}$, which is about one order of magnitude smaller than the ones mentioned in $[18,19]$. This much smaller oscillation amplitude might be due to the particular interaction in the capillary tube, as the experiments reporting larger amplitudes were all performed in gas jets.

\section{Simulations and discussion}

Simulations were carried out in order to better understand the interaction processes [14] for the low laser intensity and low electron density used in the experiment, where one would not expect trapping and acceleration of electrons to occur and consequently no betatron X-rays to be produced. Particle-in-cell (PIC) simulations were performed using the electromagnetic code CALDER-CIRC [21]. To model the propagation of the laser pulse along the capillary tube, a dielectric boundary condition was added to the code. Detailed results of these simulations are presented in [14]. Additional simulations results are shown in this section. They were performed for a 35 fs laser pulse with a radial intensity profile fitting the $\theta$-averaged experimental profile, and a peak normalized laser amplitude of 0.6. The focal plane was located at the capillary entrance. The capillary diameter was $150 \mu \mathrm{m}$, its length $20 \mathrm{~m}$. A ramp of $400 \mu \mathrm{m}$ increased the electron density from 0 to $n_{e}$, with $n_{e}$ being the electron density inside the capillary. Simulations were carried out for three different electron densities $n_{e}=5,6.5$ and $8 \times 10^{18} \mathrm{~cm}^{-3}$.

An example of results is shown in Fig. 8 for $n_{e}=$ $5 \times 10^{18} \mathrm{~cm}^{-3}$. The solid black curve shows the evolution of the normalized laser amplitude along the axis of the 


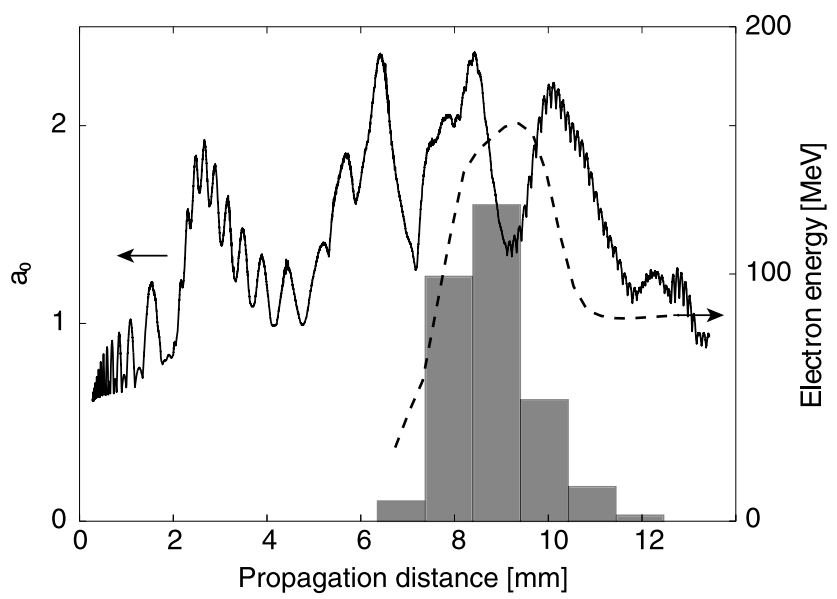

Fig. 8 Simulation of the interaction in the capillary tube using the PIC code CALDER-CIRC. The solid black curve shows the evolution of the laser intensity along the capillary length. The dashed line shows the energy of the first electron bunch. The corresponding X-ray emission is shown by the grey bars, with the total X-ray energy plotted in arbitrary units. The maximum of the X-ray emission coincides with the maximum energy of the electrons

capillary tube. The beam undergoes a slow process of selffocusing, reaching a maximum amplitude of $a_{0}=2.5$ after a propagation of $6.4 \mathrm{~mm}$ (i.e. the intensity has increased by a factor 17). This relatively modest amplitude is nevertheless large enough to produce self-injection of electrons, mainly into the first bucket of the wakefield, even at such low density. The capillary confines the energy surrounding the main peak of the focal spot and contributes to maintaining the beam self-focused $2-3 \mathrm{~mm}$ longer than without the capillary. The simulations show that the evolution of the laser intensity depends strongly on capillary diameter, initial laser intensity, and electron density, as the mode propagation is quite sensitive to these parameters. This is consistent with our experimental observations, as shown in Figs. 4(a) and 7, where the optimum density and the position of the trapping point depend strongly on these parameters.

After trapping, the electron bunch is accelerated by the plasma wave in the capillary. Simulations show that the electrons reach dephasing, as illustrated by the dashed line in Fig. 8 showing the energy of the first electron bunch. This is also consistent with the experimental electron data (see Fig. 4(b)). At this low density, the dephasing length is $\sim 5 \mathrm{~mm}$ but dephasing is still reached inside the capillary. The extracted electron bunch therefore presents a wide energy spectrum, similar to the experimental spectrum shown in Fig. 3(b). In some cases, the peaks in the simulated electron spectra are more pronounced, but the quasi-monoenergetic features observed experimentally, such as the one shown in Fig. 3(a), are not reproduced in the simulations. Mono-energetic spectra could result from a strongly asymmetric self-injection of electrons, originated in beam asym- metry and eventually associated hosing. Further studies taking into account asymmetrical features are needed to test these hypotheses.

The X-ray spectra generated by accelerated electrons, obtained in the simulations, are found to be synchrotron-like, with a critical energy of about $1 \mathrm{keV}$ in fair agreement with experimental data. The total $\mathrm{X}$-ray energy (integrated from 0 to $10 \mathrm{keV}$ ) is also calculated and shown by the grey bars in Fig. 8. It is clearly seen that most of the X-rays are generated in the region where electrons reach their maximum energy. The emitted power scales with $\gamma^{4}$ [11] and, therefore, the $\mathrm{X}$-ray emission is maximum when the electrons have their maximum energy. The spatial profile is calculated with the assumption that the X-rays are absorbed at the capillary wall. The simulated results are very close to the experimental ones, as seen for example in Fig. 5(c). In the simulations, the radius of the X-ray beam is also found to vary as a function of the electron density. This agrees with our explanation that the diameter of the X-ray beam on the detector gives us an indication about the position of the trapping point. For $n_{e}=8 \times 10^{18} \mathrm{~cm}^{-3}$, the simulations shows that the electrons are trapped early $(\sim 3 \mathrm{~mm})$ and the divergence of the $\mathrm{X}$-ray beam is $9.4 \mathrm{mrad}$. For $n_{e}=5 \times 10^{18} \mathrm{~cm}^{-3}$, electrons are trapped later $(\sim 7 \mathrm{~mm})$, and the divergence of the X-ray beam is therefore much bigger $(13.6 \mathrm{mrad})$. The trapping position estimated from the simulation results fairly agree with the experimental determination.

\section{Conclusion}

Experimental data show that inside dielectric capillaries trapping and acceleration of electrons is possible at lower laser intensities than in gas jets, where in both cases the laser pulse interacts with a neutral gas homogeneous in the transverse direction. It was found that the capillary confines energy located outside the main peak in the focal plane and contributes to the beam self-focusing over longer distances, thus allowing the pulse to evolve enough to reach the threshold for trapping and acceleration of electrons. The interaction was found to depend strongly, and in a complex way, on parameters such as plasma electron density, capillary diameter, and laser intensity. This has been investigated experimentally, both by observing the accelerated electron beam directly and by analysing betatron X-ray radiation as an additional diagnostic of the interaction. Simulations are in good agreement with experimental results. The work presented here shows that the interaction in a capillary tube is different from the interaction in more conventional targets, such as gas jets. This suggests that this kind of structures has interesting properties for electron acceleration. In order to explore the potential of these novel targets for electron acceleration, further studies are required. 
Acknowledgements We acknowledge the support of the European Community-NEST Activity under the FP6 Structuring the European Research Area programme (project EuroLEAP, contract 028514), Marie Curie Early Stage Training Site MAXLAS (MEST-CT-2005020356), the Lund University X-ray Center (LUXC), the Swedish Research Council (including the Linné grant to the LLC), the Knut and Alice Wallenberg Foundation, the RTRA Triangle de la Physique, and the French Agence Nationale de la Recherche. We also thank the referees for their constructive comments.

Open Access This article is distributed under the terms of the Creative Commons Attribution Noncommercial License which permits any noncommercial use, distribution, and reproduction in any medium, provided the original author(s) and source are credited.

\section{References}

1. S.P.D. Mangles, C.D. Murphy, Z. Najmudin, A.G.R. Thomas, J.L. Collier, A.E. Dangor, E.J. Divall, P.S. Foster, J.G. Gallacher, C.J. Hooker, D.A. Jaroszynski, A.J. Langley, W.B. Mori, P.A. Norreys, F.S. Tsung, R. Viskup, B.R. Walton, K. Krushelnick, Nature (London) 431, 535 (2004)

2. C.G.R. Geddes, Cs. Toth, J. van Tilborg, E. Esarey, C.B. Schroeder, D. Bruhwiler, C. Nieter, J. Cary, W.P. Leemans, Nature (London) 431, 538 (2004)

3. J. Faure, Y. Glinec, A. Pukhov, S. Kiselev, S. Gordienko, E. Lefebvre, J.-P. Rousseau, F. Burgy, V. Malka, Nature (London) 431, 541 (2004)

4. S.P.D. Mangles, A.G.R. Thomas, M.C. Kaluza, O. Lundh, F. Lindau, A. Persson, F.S. Tsung, Z. Najmudin, W.B. Mori, C.-G. Wahlström, K. Krushelnick, Phys. Rev. Lett. 96, 215001 (2006)

5. J. Osterhoff, A. Popp, Zs. Major, B. Marx, T.P. Rowlands-Rees, M. Fuchs, M. Geissler, R. Hörlein, B. Hidding, S. Becker, E.A. Peralta, U. Schramm, F. Grüner, D. Habs, F. Krausz, S.M. Hooker, S. Karsch, Phys. Rev. Lett. 101, 085002 (2008)

6. W.P. Leemans, B. Nagler, A.J. Gonsalves, Cs. Tóth, K. Nakamura, C.G.R. Geddes, E. Esarey, C.B. Schroeder, S.M. Hooker, Nat. Phys. 2, 696 (2006)

7. B. Cros, C. Courtois, G. Matthieussent, A. Di Bernardo, D. Batani, N. Andreev, S. Kuznetsov, Phys. Rev. E 65, 026405 (2002)
8. F. Dorchies, J.R. Marquès, B. Cros, G. Matthieussent, C. Courtois, T. Vélikoroussov, P. Audebert, J.P. Geindre, S. Rebibo, G. Hamoniaux, F. Amiranoff, Phys. Rev. Lett. 82, 4655 (1999)

9. F. Wojda, K. Cassou, G. Genoud, M. Burza, Y. Glinec, O. Lundh, A. Persson, G. Vieux, E. Brunetti, R.P. Shanks, D. Jaroszynski, N.E. Andreev, C.-G. Wahlström, B. Cros, Phys. Rev. E 80, 066403 (2009)

10. A. Rousse, K.T. Phuoc, R. Shah, A. Pukhov, E. Lefebvre, V. Malka, S. Kiselev, F. Burgy, J.-P. Rousseau, D. Umstadter, D. Hulin, Phys. Rev. Lett. 93, 135005 (2004)

11. E. Esarey, B. Shadwick, P. Catravas, W. Leemans, Phys. Rev. E 65, 056505 (2002)

12. J.D. Jackson, in Classical Electrodynamics, 3rd edn. (Wiley, New York, 1975), Chap. 14

13. G. Genoud, M. Burza, A. Persson, F. Wojda, C.-G. Wahlström, Rev. Sci. Instrum. 82, 033102 (2011)

14. H.E. Ferrari, A.F. Lifschitz, B. Cros, Plasma Phys. Control. Fusion 53, 014005 (2010)

15. S. Kneip, S.R. Nagel, C. Bellei, N. Bourgeois, A.E. Dangor, A. Gopal, R. Heathcote, S.P.D. Mangles, J.R. Marquès, A. Maksimchuk, P.M. Nilson, K.T. Phuoc, S. Reed, M. Tzoufras, F.S. Tsung, L. Willingale, W.B. Mori, A. Rousse, K. Krushelnick, Z. Najmudin, Phys. Rev. Lett. 100, 105006 (2008)

16. S. Kneip, C. McGuffey, J.L. Martins, S.F. Martins, C. Bellei, V. Chvykov, F. Dollar, R. Fonseca, C. Huntington, G. Kalintchenko, A. Maksimchuk, S.P.D. Mangles, T. Matsuoka, S.R. Nagel, C.A.J. Palmer, J. Schreiber, K.T. Phuoc, A.G.R. Thomas, V. Yanovsky, L.O. Silva, K. Krushelnick, Z. Najmudin, Nat. Phys. 6, 980 (2010)

17. R.C. Shah, F. Albert, K.T. Phuoc, O. Shevchenko, D. Boschetto, A. Pukhov, S. Kiselev, F. Burgy, J.-P. Rousseau, A. Rousse, Phys. Rev. E 74, 045401 (2006)

18. K.T. Phuoc, S. Corde, R. Shah, F. Albert, R. Fitour, J.-P. Rousseau, F. Burgy, B. Mercier, A. Rousse, Phys. Rev. Lett. 97, 225002 (2006)

19. S.P.D. Mangles, G. Genoud, S. Kneip, M. Burza, K. Cassou, B. Cros, N.P. Dover, C. Kamperidis, Z. Najmudin, A. Persson, J. Schreiber, F. Wojda, C.-G. Wahlström, Appl. Phys. Lett. 95, 181106 (2009)

20. K. Ta Phuoc, S. Corde, R. Fitour, R. Shah, F. Albert, J.-P. Rousseau, F. Burgy, A. Rousse, V. Seredov, A. Pukhov, Phys. Plasmas 15, 073106 (2008)

21. A.F. Lifschitz, X. Davoine, E. Lefebvre, J. Faure, C. Rechatin, V. Malka, J. Comput. Phys. 228, 1803 (2009) 